\title{
True colour Denisyuk-type hologram recording in Bayfol HX self-developing photopolymer
}

\author{
Irene Vázquez-Martín ${ }^{\mathrm{a}}$, Marina Gómez-Climente ${ }^{\mathrm{a}}$, Julia Marín-Sáez ${ }^{\mathrm{b}}$, M. Victoria Collados ${ }^{\mathrm{a}}$, \\ Jesús Atencia ${ }^{\mathrm{a}^{*}}$ \\ ${ }^{a}$ Departamento de Física Aplicada, Instituto de Investigación en Ingeniería de Aragón (I3A), \\ Universidad de Zaragoza, Facultad de Ciencias, E-50009 Zaragoza, Spain; \\ ${ }^{\mathrm{b}}$ Departamento de Medio Ambiente, Sección de Física Aplicada. Escuela Politécnica, Universitat de \\ Lleida, E-25001 Lleida, Spain
}

\begin{abstract}
True colour Denisyuk-type hologram recording of diffusing objects in Bayfol ${ }^{\circledR}$ HX 102 self-developing photopolymer has been studied. In a first stage, monochromatic Denisyuk holograms of a standard white diffuser (Spectralon) have been recorded using lasers with wavelengths 442, 532 and $633 \mathrm{~nm}$ to determine the optimum exposure that gives maximum efficiency. The recording of holograms from a diffusing object has the particularity that intermodulation noise due to interference between waves arriving from different object points reduces effective index modulation. A maximum effective efficiency of $80 \%$ has been reached for monochromatic recording. In a second stage, a set of experiments has been carried out to determine the adequate relation of exposure for the recording of a Denisyuk hologram of the standard white diffuser with the three lasers simultaneously to get the maximum efficiency for each wavelength. With the determined optimal exposure, a hologram of a polychromatic diffusing object has been recorded, obtaining a good visual coincidence between hologram and original object.
\end{abstract}

Keywords: Volume holography, photopolymer, colour hologram, reflection holography, Denisyuk hologram.

\section{INTRODUCTION}

Realistic true colour reproduction is one of the relevant objectives of holography. The first colour holograms were transmission type, and they were recorded using three lasers of different wavelengths with different directions of the reference beams to avoid overlaps ${ }^{1}$. This technique requires a complicated lighting system for the reconstruction of the image, since it is necessary to use the three lasers in directions similar to those of construction.

Since reflection holograms can be reconstructed with white light, this is the type currently used for the recording of colour holograms, especially in Denisyuk's single beam configuration due to its simplicity of recording and reconstruction ${ }^{2}$; thus this is the technique chosen for this work. Numerous studies have been conducted to optimize the recording of colour Denisyuk holograms in silver halide materials. In particular, Bjelkhagen's work ${ }^{3}$ with SilverCross panchromatic silver halide emulsion, in which realistic holograms have been achieved.

Recently, the German company Covestro (formerly Bayer MaterialScience) has developed a panchromatic photopolymer, Bayfol® HX 102, which allows the recording of colour holograms ${ }^{4}$. It is a self-developing photopolymer; the polymerization (and so, the hologram formation) begins when it is illuminated with the recording beams, so recording with different wavelengths requires simultaneous or sequential exposure ${ }^{5}$. Photopolymers have become very popular in holography since some do not require any post-exposure treatment or, in the worst case, only require dry processing. With this material it is possible to obtain high diffraction efficiencies and high stability after recording, with relatively low cost. On the other hand, they are less sensitive than silver halide materials and they cannot be stored unexposed for long periods of time.

The holographic recording requires coherent, and therefore monochromatic light sources. The number of wavelengths necessary for reliable colour recording has been theoretically studied by Bjelkhagen et al. ${ }^{3}$, concluding that, although

*atencia@unizar.es 
using four or more wavelengths provides a more precise colour reconstruction, with three lasers with wavelengths around 466, 545 and $610 \mathrm{~nm}$ the error in colour reproduction is tolerable. While increasing the number of lasers improves colour reproduction, it will also increase the complexity and cost of the recording set-up. For this reason, a three laser configuration is used in this work.

The main objective of this study is the calibration of the material Bayfol ${ }^{\circledR}$ HX 102 for the recording of colour Denisyuk type holograms from diffusing objects. First, the calibration was done with a standard white diffuser object for each of the three separate recording wavelengths $(442 \mathrm{~nm}, 532 \mathrm{~nm}$ and $633 \mathrm{~nm})$. Then, based on the data obtained with the three wavelengths separately, the intensity of each of the lasers was adjusted to perform the calibration by exposing the material simultaneously to the three wavelengths. Finally, the hologram of a colour diffusing object was recorded.

\section{EXPERIMENTAL SETUP}

\subsection{Holographic material: Bayfol HX 102 .}

The material used in this work was the photopolymer Bayfol ${ }^{\circledR}$ HX $102^{6}$. It is a photosensitive and self-processing polymer that serves to construct volume holograms of both reflection and transmission type. It is sensitive to wavelengths in the spectral range from $400 \mathrm{~nm}$ to $700 \mathrm{~nm}$. Additional treatments are not necessary for the formation of the hologram. The holographic sheet consists of a polycarbonate substrate on which a layer of photopolymer of thickness $d=16 \pm 2 \mu \mathrm{m}$ is deposited.

The surfaces of the substrate and the photopolymer are covered by a protective film, which must be removed before the hologram recording. Pre-exposure sample handling should be done in a dark room, although a safety light of a wavelength for which the material is not sensitive can be used. Figure 1 shows the transmission spectrum of the unexposed photopolymer ${ }^{6}$.

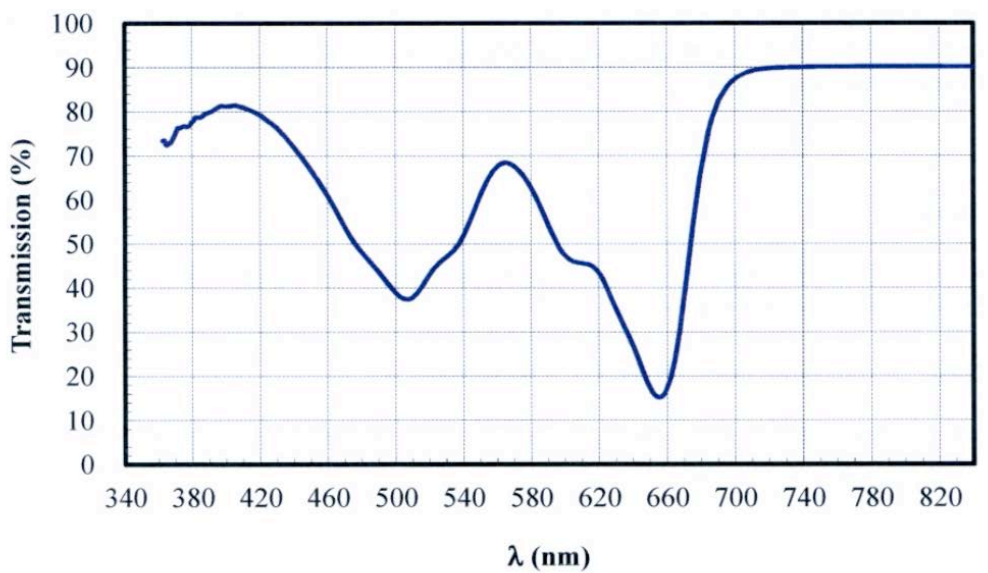

Figure 1: Transmission spectrum of the unexposed photopolymer ${ }^{6}$.

The maximum absorption $(85 \%)$ is found for approximately $655 \mathrm{~nm}$, and a second maximum $(62 \%)$ is around $510 \mathrm{~nm}$. In contrast, there is a minimum $(30 \%)$ around $570 \mathrm{~nm}$, so the use of a yellow security light is recommended in the specification sheet of the material. For the experiments a $590 \mathrm{~nm}$ LuxeonRebel LT1012 LED light source was used, which must be located at least $50 \mathrm{~cm}$ away from the material.

Once the material is exposed in the holographic recording, the monomer begins to polymerize in the regions where it receives sufficient light. Thus, changes in exposure are associated with gradients of monomer concentration due to changes in polymerization. In the regions where the polymerization has occurred, the monomer concentration decreases, causing a concentration gradient and thus its diffusion from the high concentration to the low concentration regions. This process takes about 5 minutes. At this stage, refraction index variations are obtained in the material and the hologram can already be seen in the photopolymer. Finally, a photocuring process is required for fixing the hologram (polymerization of the remaining monomer and bleaching of the dye), which is done by illuminating the hologram with a white light led lamp of $50 \mathrm{~W}$ for 25 minutes $^{7}$. 
There are no significant changes in material thickness during the process. According to the manufacturer, the variation is less than $1.4 \%{ }^{6}$. This feature makes the Bayfol ${ }^{\circledR}$ HX 102 ideal for recording holograms in real colour. The maximum index modulation achievable with this material in reflection gratings is $\Delta n \cong 0.033^{4}$. Smaller effective modulation is expected for the recording of diffuser objects due to intermodulation noise due to interference between object points.

Bayfol HX® 102 sheets have a slight curvature that allows the identification of the surface of the photopolymer and the substrate. The convex part corresponds to the photopolymer. Before recording, the samples can be directly adhered, to a $3.9 \mathrm{~mm}$ thick glass thanks to the viscosity of the photopolymer. Prior to this step, the glass must be carefully cleaned to provide as few dust particles as possible. The presence of these particles when adhering the photopolymer to the glass causes the existence of bubbles that can produce vibrations in that zone of the photopolymer, averaging the interferential figure and so avoiding hologram formation.

\subsection{Recording setup.}

Three lasers with wavelengths close to the optimum ones ${ }^{3}$, shown in Table 1, have been chosen.

Table 1: Lasers used in the recordings, optimal wavelengths ${ }^{3}$ and relative error.

\begin{tabular}{|c|c|c|c|}
\hline Laser & Kimmon IK4171I-G & DPSS Oxxius LMX & Uniphase 1145P \\
\hline Power $(\mathbf{m W})$ & 150 & 100 & 35 \\
\hline $\boldsymbol{\lambda} \mathbf{( n m )}$ & 442 & 532 & 633 \\
\hline Optimal $\boldsymbol{\lambda}$ & 466 & 545 & 610 \\
\hline$\Delta \boldsymbol{\lambda} \boldsymbol{\lambda} \mathbf{\lambda ( \% )}$ & 5 & 2 & 4 \\
\hline
\end{tabular}

In figure 2, these wavelengths are shown on the CIE diagram. The lines marked in black enclose the colours that can be reproduced with the three wavelengths used in this work.

The experimental setup used for the recording of Denisyuk type holograms is shown in Figure 3. The three laser beams, with linear polarization in a direction perpendicular to the plane of the figure, are combined by dichroic mirrors. Neutral reflective filters were placed in the optical paths of the green and the blue lasers to adjust the intensity. Each shutter permits independent exposure time control for each wavelength. The three combined lasers pass through a microscope objective. In the focus of the objective a pinhole is placed to spatially filter the beam, eliminating noise due to dust particles or other imperfections. Finally the three combined and expanded beams illuminate the photopolymer and the object. The diameter of the blue $442 \mathrm{~nm}$ laser beam is approximately three times smaller than the green and red laser beams, therefore a $3 x$ Galileo telescope is introduced into the blue beam so that the three lasers illuminate a similar area, large enough to cover the object completely.

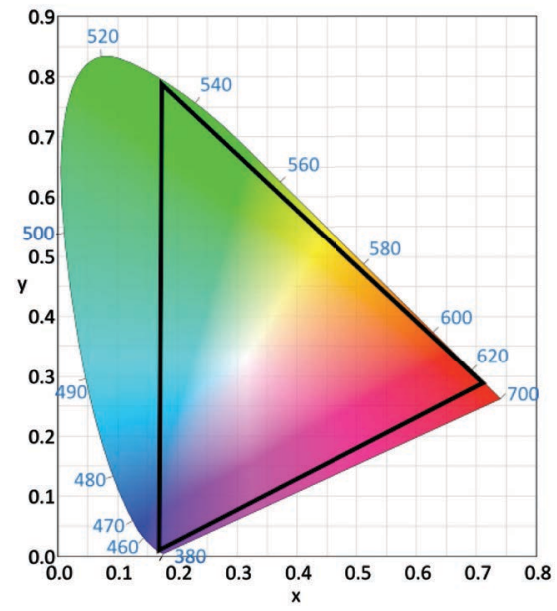

Figure 2: CIE diagram of 1931, on which the triangle formed by the wavelengths of the lasers used in the work is shown. 


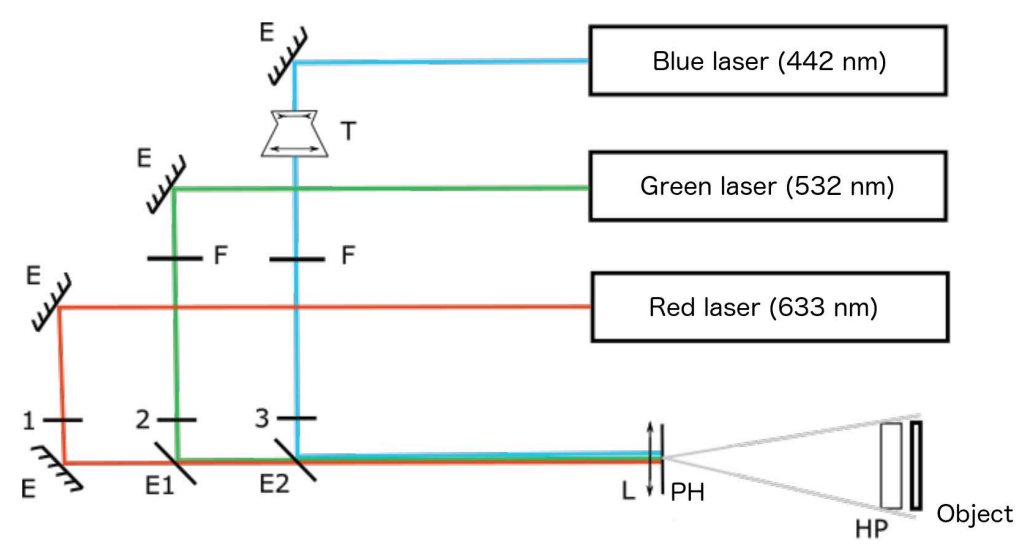

Figure 3: Recording setup diagram. $\mathrm{T}$ is the telescope, $\mathrm{F}$ the neutral reflective filters, $\mathrm{E}$ the broadband mirrors, 1, 2 and 3 are shutters for each laser, E1 and E2 are the dichroic mirrors, L the objective lens, PH the pinhole and HP the photopolymer.

The object used for the calibration is a flat plate of Spectralon ${ }^{\circledR}$, a plastic material of very high diffuse reflectance in the ultraviolet, visible and near infrared ranges. It has a highly Lambertian behaviour, so it will be used as a perfect diffuser target. The glass plate with the photopolymer is firmly attached to the Spectralon plate, with the glass in contact with the object. Both object and photopolymer are placed tilted with respect to the plane of Figure 3, forming Brewster's angle with the reference beam so that the reflected beam is cancelled, thus avoiding possible interferences in both glass surfaces and the photopolymer that can cause spurious holograms.

The use of a perfect diffuser results in loss of intensity of the object wave that may interfere with the reference wave. This material diffuses completely depolarized light, whereby only the component on the polarization direction of the incident wave interferes; that is, at most $50 \%$ of the total object intensity. Another factor that attenuates the intensity of the object beam is the absorption of the photopolymer, because the reference beam passes through it before reaching the object. Since the intensity of the object beam cannot be measured in the setup, the calibration will be performed based on the reference beam exposure.

\section{CALIBRATION}

\subsection{Monochromatic calibration.}

The first step is to determine the exposure that produces maximum efficiency when the material is exposed to monochromatic radiation. The calibration is performed separately for each of the three wavelengths that we will use later to record colour holograms. To do this, different samples are exposed varying the exposure time, with the reference beam intensity $I_{i}$ given in table 2 . The relative efficiency of the recorded volume holograms is determined as:

$$
\eta=\frac{\phi_{+1}}{\phi_{0}+\phi_{+1}}
$$

where $\phi_{+1}$ is the diffracted +1 order flux and $\phi_{0}$ is 0 -diffracter order flux. As in volume holograms only 0 -order and +1 order are diffracted, $\phi_{+1}$ y $\phi_{0}$ are measured from the 0 -order spectrum by means of an Ocean Optics USB2000 spectrometer as shown in Figure 4.

Table 2: Incident intensity, maximum efficiency and exposure required to obtain $99 \%$ of the maximum efficiency for each of the three wavelengths.

\begin{tabular}{|c|c|c|c|}
\hline$\lambda(\mathbf{n m})$ & 442 & 532 & 633 \\
\hline $\boldsymbol{I}_{\boldsymbol{i}}\left(\mathbf{m W} / \mathbf{c m}^{\mathbf{2}}\right)$ & 0,4 & 0,5 & 0,1 \\
\hline$\eta_{\max } \mathbf{( \% )}$ & 60 & 80 & 63 \\
\hline $\boldsymbol{E}$ for $\mathbf{0 . 9 9} \eta_{\max }\left(\mathbf{m J} / \mathbf{c m}^{\mathbf{2}}\right)$ & 25,6 & 8,4 & 8,8 \\
\hline
\end{tabular}




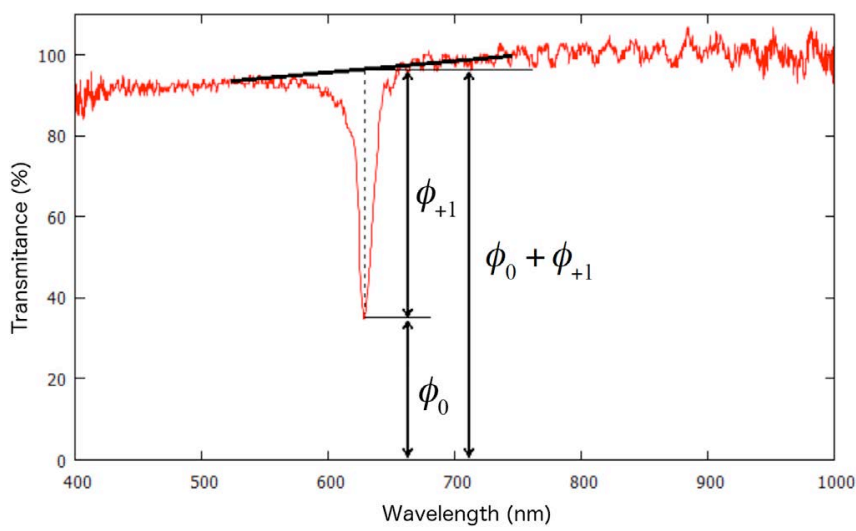

Figure 4: Characteristic transmitted spectrum for a reflection diffusing-object hologram recorded with $633 \mathrm{~nm}$ laser light.

Figure 5 shows the efficiency values obtained as a function of the exposure, together with the theoretical dependence expected for reflection volume holograms. It can be observed in the three graphs the presence of a threshold exposure, below which the energy is not enough to initiate the polymerization. As soon as the energy is high enough, the efficiency grows rapidly until it reaches a saturation zone. Since the maximum efficiency is asymptotically achieved when the exposure tends to infinity, we will consider as optimum exposure that for which about $99 \%$ of the maximum efficiency is achieved. The data obtained are shown in Table 2.

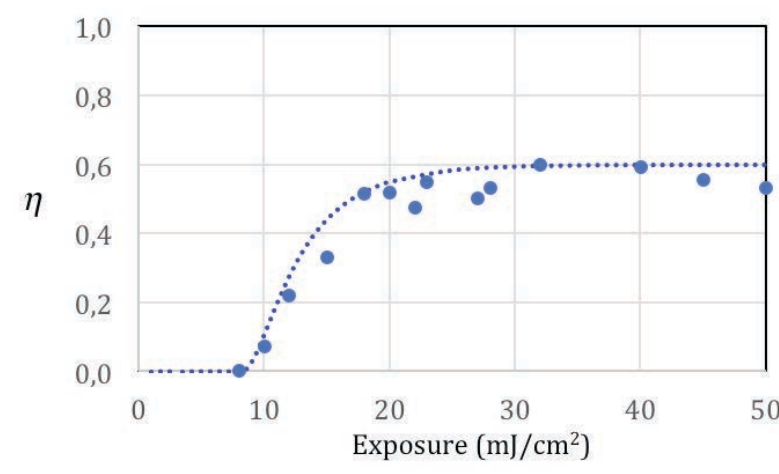

(a)

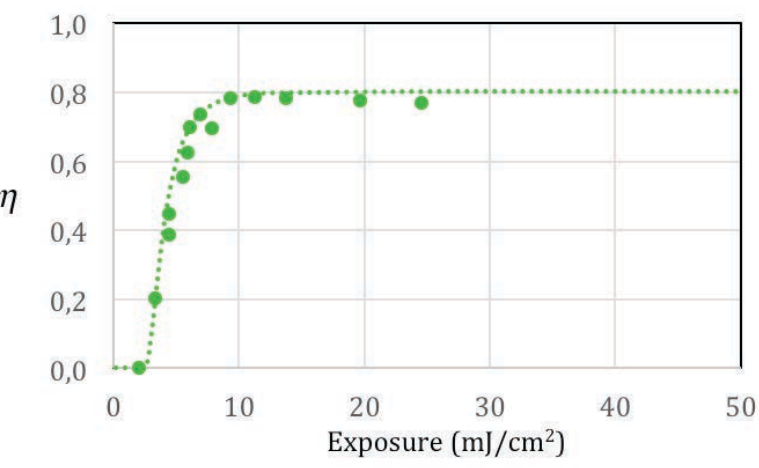

(b)

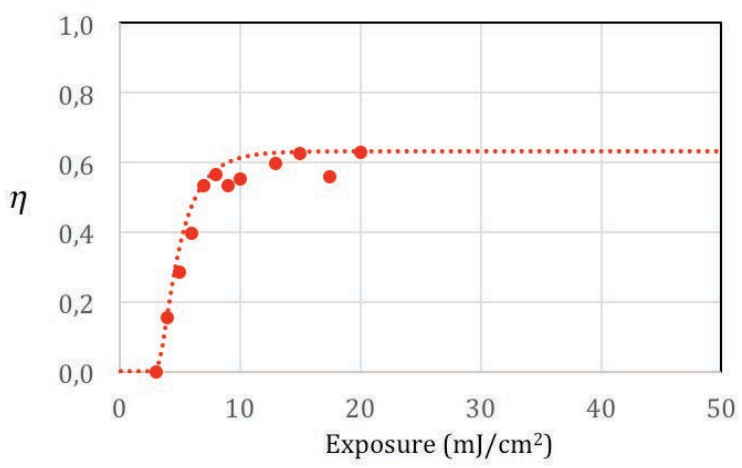

(c)

Figure 5: Efficiency as a function of exposure for recording wavelength, $442 \mathrm{~nm}$ (a), $532 \mathrm{~nm}$ (b) and $633 \mathrm{~nm}$ (c). The theoretical dependence for reflection volume holograms is represented in a dashed line. 


\subsection{Polychromatic calibration.}

When the photopolymer is simultaneously exposed to the three lasers, an independent hologram is recorded with each wavelength. It is expected that the efficiency of each hologram is lower than in monochromatic recording because index modulation must be distributed between the three holograms.

For the polychromatic recording, two exposure methods have been considered:

a) Simultaneous exposure with same exposure time; to do this, the laser intensities are adjusted using neutral density filters ( $\mathrm{F}$ in figure 3 ) to achieve the proper exposures.

b) Sequential exposure. The material is exposed independently for each wavelength, making series of short sequential exposures ${ }^{5}$.

\subsubsection{Simultaneous exposure with same exposure time.}

In this case the hologram is recorded illuminating with the three wavelengths simultaneously and with the same exposure time. Since the exposure values to achieve the maximum efficiency are different for each wavelength (Table 2 and Figure 5), the reference beam intensity of each wavelength must be adjusted to get the same exposure time for the three wavelenghts. To do this it is necessary to decrease the intensity of the green and blue lasers to match the exposure time needed with the red laser. For this purpose, neutral filters have been used, obtaining an intensity ratio given in Table 3.

Table 3: Incident intensity and maximum efficiency obtained with simultaneous exposure for each of the three wavelengths.

\begin{tabular}{|c|c|c|c|}
\hline $\boldsymbol{\lambda} \mathbf{( n m )}$ & 442 & 532 & 633 \\
\hline $\boldsymbol{I}_{\mathbf{i}} \mathbf{( m W / \mathbf { c m } ^ { 2 } )}$ & 0,32 & 0,10 & 0,10 \\
\hline $\boldsymbol{\eta}_{\max } \mathbf{( \% )}$ & 14,9 & 24,2 & 16,3 \\
\hline
\end{tabular}

Figure 6 shows the efficiency values measured at each wavelength as a function of the exposure, together with the theoretical dependence expected for reflection volume holograms. Maximum values of efficiency obtained are given in Table 3 .

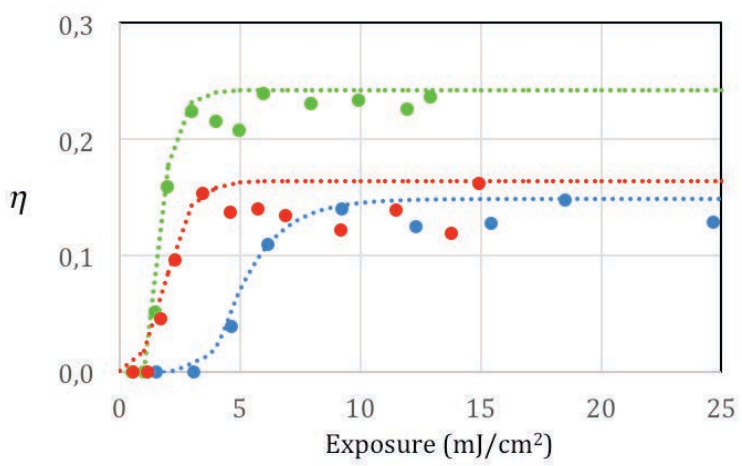

Figure 6: Efficiency as a function of simultaneous exposure for reconstruction wavelength, $442 \mathrm{~nm}$ (blue markers), $532 \mathrm{~nm}$ (green markers) and $633 \mathrm{~nm}$ (red markers). The theoretical dependence for reflection volume holograms is represented with dashed lines.

In figure 6 it is possible to see that the three holograms recorded simultaneously (one for each color) compete with each other in the distribution of the index modulation, obtaining a greater efficiency for the green. In order to match the efficiency of the three wavelengths, as well as to improve such efficiency, another exposure method is tested.

\subsubsection{Sequential exposure.}

In the work of Piao et al. ${ }^{5}$ a sequential exposure method is used to improve the efficiency of color transmission holograms. That method consists in exposing the material alternately to each laser for short periods of time, with the set of exposures repeating a determined number of cycles. 
This method is applied to our setup using shutters that independently control the exposure time with each laser (shutters 1, 2, and 3 shown in Figure 3). To avoid instabilities due to high shutter frequencies, $t \geq 2 \mathrm{~s}$ is taken as the minimum shutter interval. The intensities used are the same shown in Table 2. A ratio of time intervals proportional to the required times in each wavelength to reach $99 \%$ of the maximum efficiency for each of the holograms (shown in Table 2) is chosen. The selected time intervals are shown in Table 4.

Table 4: Time exposure intervals and maximum efficiency obtained with sequential exposure for each of the three wavelengths.

\begin{tabular}{|c|c|c|c|}
\hline $\boldsymbol{\lambda} \mathbf{( n m )}$ & 442 & 532 & 633 \\
\hline $\boldsymbol{t} \mathbf{( s )}$ & 8,0 & 2,0 & 9,0 \\
\hline$\eta_{\max } \mathbf{( \% )}$ & 22,5 & 14,5 & 24,7 \\
\hline
\end{tabular}

This cycle is repeated as many times as necessary to get the desired exposure. Figure 7 shows the efficiency for each wavelength as a function of exposure in holograms recorded with sequential exposure. Maximum values of efficiency obtained are given in Table 4. We can see that the efficiencies are higher than in simultaneous exposure, but in this case the efficiency of green wavelenght is slightly lower.

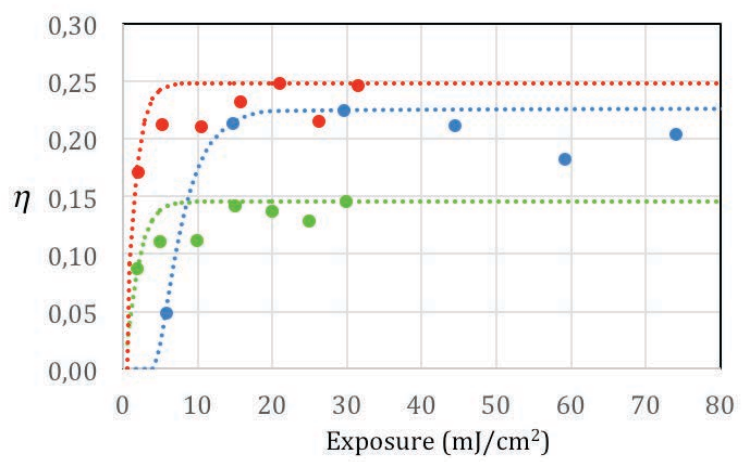

Figure 7: Efficiency as a function of exposure (sequential) for reconstruction wavelength, $442 \mathrm{~nm}$ (blue markers), $532 \mathrm{~nm}$ (green markers) and $633 \mathrm{~nm}$ (red markers). The theoretical dependence for reflection volume holograms is represented with dashed lines.

To illustrate the utility of the obtained results, a true colour hologram is recorded using as an object a figure painted with different colors instead of the white Spectralon diffuser. Figure 8 shows a photograph of the object and a photograph of the true colour hologram obtained, both illuminated with the same white light.

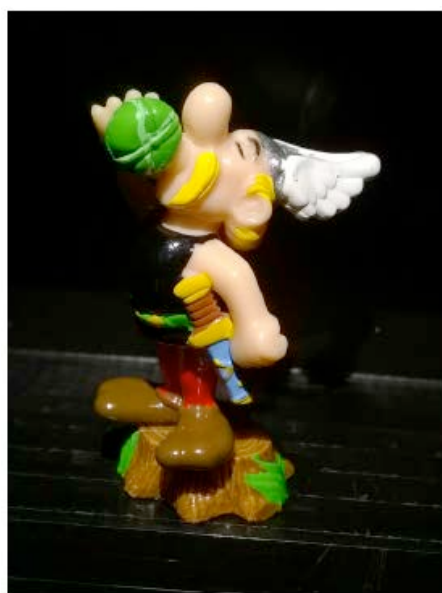

(a)

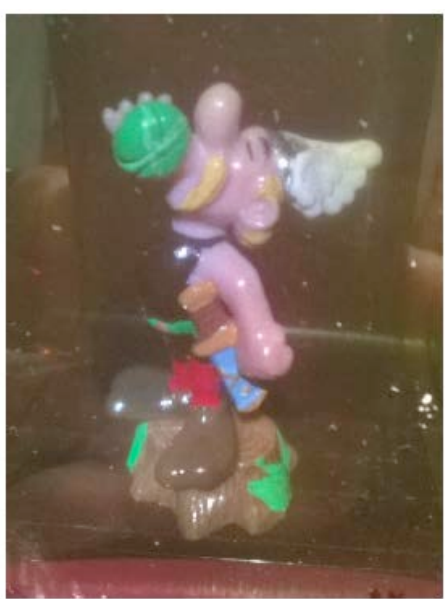

(b)

Figure 8: (a) Photography of the figure used as object (b) Photography or the true colour hologram obtained. 


\section{CONCLUSIONS.}

The true colour Denisyuk-type hologram recording of diffusing objects in Bayfol HX 102 self-developing photopolymer has been demonstrated.

In the monochromatic recording, maximum efficiencies of $60 \%$ for $442 \mathrm{~nm}, 80 \%$ for $532 \mathrm{~nm}$ and $63 \%$ for $633 \mathrm{~nm}$ were achieved.

Two methods of color recording have been tested: simultaneous exposure with same exposure time or sequential exposure. With the first one, maximum efficiencies of $10,9 \%$ for $442 \mathrm{~nm}, 15,9 \%$ for $532 \mathrm{~nm}$ and $9,6 \%$ for $633 \mathrm{~nm}$ were achieved. With the second one, a better result was obtained: $22,5 \%$ for $442 \mathrm{~nm}, 14,5 \%$ for $532 \mathrm{~nm}$ and $24,7 \%$ for $633 \mathrm{~nm}$

More experiments with different ratio of time intervals or different exposure method can be carried out to match and maximize the efficiencies for each wavelength.

\section{REFERENCES}

[1] Bjelkhagen, H. and Brotherton-Ratcliffe, D., [Ultra-Realistic Imaging: Advanced techniques in analogue and digital colour holography], CRC Press-Taylor and Francis Group, Boca Raton (2013).

[2] Denisyuk, Y. N., "On the reproduction of the optical properties of an object by the wave field of its scattered radiation," Optical Spectroscopy 14, 279-284 (1963).

[3] Bjelkhagen, H. I. and Mirlis, E., "Color holography to produce highly realistic three-dimensional images," Applied Optics 47, 123-133 (2008).

[4] Berneth, H., Bruder, F. K., Fäcke, T., Hagen, R., Hönel, D., Jurbergs, D., Rölle, T. and Weiser, M. S., "Holographic recording aspects of high-resolution Bayfol® HX photopolymer," Proc. SPIE 7957, 79570H (2011).

[5] Piao, M. L., Kwon, K. C., Kang, H. J., Lee, K. Y. and Kim, N., "Full-color holographic diffuser using timescheduled iterative exposure," Applied Optics 54, $5252-5259$ (2015).

[6] Bayer MaterialScience, "Bayfol® HX 102 film. Preliminary product information,"

[7] Marín-Sáez, J., Atencia, J., Chemisana, D. and Collados, M. V., "Characterization of volume holographic optical elements recorded in Bayfol HX photopolymer for solar photovoltaic applications," Optics Express 24(6), 720-730 (2016). 